Carlos Frederico da Silva Crespo

\begin{abstract}
AVALIAÇÃO DO IMPACTO ECONÔMICO DE UM PROJETO DE PESQUISA E DESENVOLVIMENTO NO VALOR DE UMA PLANTA "GAS-TO-LIQUID" USANDO A TEORIA DAS OPÇÕES REAIS
\end{abstract}

Tese de Doutorado

Tese apresentada como requisito parcial para obtenção do título de Doutor em Engenharia Industrial pelo Programa de Pós-Graduação em Engenharia de Produção da PUC-Rio.

Orientador: José Paulo Teixeira

Rio de Janeiro

Julho de 2008 


\section{AVALIAÇÃO DO IMPACTO ECONÔMICO DE UM PROJETO DE PESQUISA E DESENVOLVIMENTO NO VALOR DE UMA PLANTA “GAS-TO-LIQUID” USANDO A TEORIA DAS OPÇÕES REAIS}

Tese apresentada como requisito parcial para a obtenção do título de Doutor pelo Programa de Pós-Graduação em Engenharia de Produção da PUC-Rio. Aprovada pela Comissão Examinadora abaixo assinada.

Prof. José Paulo Teixeira Orientador

Departamento de Engenharia Industrial - PUC-Rio

Prof. Leonardo Pereira Santiago

UFMG

Prof. Carlos Patrício Samanez

Departamento de Engenharia Industrial - PUC-Rio

Prof. Fabio Rodrigo Siqueira Batista

Departamento de Engenharia Industrial - PUC-Rio

Prof. Luiz Eduardo Teixeira Brandão

IAG PUC-Rio

Prof. Alexandre Zanini UFJF

Prof. José Eugenio Leal Coordenador Setorial do Centro Técnico Científico -PUC-Rio 
Todos os direitos reservados. É proibida a reprodução total ou parcial do trabalho sem autorização da universidade, do autor e do orientador.

\section{Carlos Frederico da Silva Crespo}

Graduado em Engenharia Civil pela Universidade Federal de Juiz de Fora em 1974 e Mestre em Engenharia de Produção pela PUC-Rio em agosto de 1991. De Dezembro de 1976 a junho de 1998, trabalhou na Rede Ferroviária Federal e MRS Logística. Desde janeiro de 2000 é professor Assistente da Faculdade de Economia e Administração da Universidade Federal de Juiz de Fora no Departamento de Finanças e Controladoria.

Ficha Catalográfica

Crespo, Carlos Frederico da Silva

Avaliação do impacto econômico de um projeto de pesquisa e desenvolvimento no valor de uma planta "gás-to-liquid" usando a teoria das opções reais / Carlos Frederico da Silva Crespo ; orientador: José Paulo Teixeira. 2008.

128 f. : il. ; $30 \mathrm{~cm}$

Tese (Doutorado em Engenharia Industrial)Pontifícia Universidade Católica do Rio de Janeiro, Rio de Janeiro, 2008.

Inclui bibliografia

1. Engenharia industrial - Teses. 2. Opções reais. 3. Projeto de pesquisa e desenvolvimento. 4. Simulação de Monte Carlo. 5. Programação dinâmica. 6. Avaliação de projetos. 7. Planta gas-to-liquid. I. Teixeira, José Paulo. II. Pontifícia Universidade Católica do Rio de Janeiro. Departamento de Engenharia Industrial. III. Título. 


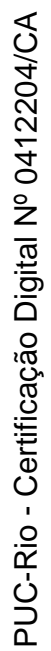

Aos meus pais José e Joanna, em memória 


\section{Agradecimentos}

Ao Professor José Paulo Teixeira que mais uma vez orientou e apoiou o meu trabalho.

Ao Professor Leonardo Santiago Pereira pela co-orientação sempre atenta e colaborativa.

À Professora Marcela Lobo Francisco, pela delicadeza e atenção a mim dispensadas no esclarecimento de dúvidas e encaminhamento de soluções.

Aos diretores da Faculdade de Economia e Administração da Universidade Federal de Juiz de Fora, pelo seu apoio inequívoco ao Programa de Qualificação Institucional da CAPES voltado para seus docentes.

Ao professor Marco Antônio Guimarães Dias pela paciência e disposição mostradas no esclarecimento de várias dúvidas durante o curso.

À Cláudia Teti, funcionária do DEI/PUC-Rio, pela paciência e competência mostradas durante todo o curso.

Aos demais funcionários e funcionárias do DEI/PUC-Rio pela postura sempre colaborativa.

À Teresa, Julia e Laura, minha família. 


\section{Resumo}

Crespo, Carlos F. S.; Teixeira, José P. Avaliação do Impacto Econômico de um projeto de Pesquisa e Desenvolvimento no valor de uma planta "Gas-to-liquids" usando a Teoria das opções Reais. Rio de Janeiro, 2008. 128p. Tese de Doutorado - Departamento de Engenharia Industrial. Pontifícia Universidade Católica do Rio da Janeiro.

Ao tomar suas decisões, o gerente se vê frente a frente com diferentes tipos de incertezas, entre elas, o tempo para se concluir um projeto. As características de inovação e ineditismo dos projetos de Pesquisa e Desenvolvimento aplicados à tecnologia reforçam ainda mais os cuidados a serem tomados com a dimensão tempo no momento da decisão, pois a penalidade pode ser até mesmo a perda de uma oportunidade valiosa de negócio. Para quantificar o impacto do esforço aplicado em um Projeto de Pesquisa e Desenvolvimento Tecnológico no valor de um empreendimento, conduzido em um contexto onde o tempo é representado por uma variável aleatória, desenvolvemos o modelo que apresentamos neste trabalho. Para tanto utilizamos a Teoria das Opções Reais, considerando que o projeto se desenvolve seqüencialmente e que pode ser abandonado em qualquer estágio de desenvolvimento. O modelo utiliza uma treliça para captar a incerteza técnica própria do desenvolvimento do desempenho da tecnologia e considera como variável aleatória o tempo de conclusão de cada uma das fases do projeto. $\mathrm{O}$ valor esperado do projeto, com a flexibilidade propiciada pela opção de abandono embutida no projeto de Pesquisa e Desenvolvimento, é calculado combinando a Simulação de Monte Carlo e a técnica da Programação Dinâmica. Aplicamos o modelo para tratar o caso de uma planta Gas-to-liquid (GTL). A função payoff do projeto de Pesquisa e Desenvolvimento foi construída a partir de diversas contribuições geradas no bojo de um convênio firmado entre o Departamento de Engenharia Industrial da PUC-RJ e a Petrobras S.A., com o objetivo de avaliar economicamente uma planta GTL.

\section{Palavras-chave}

Opções Reais; Projeto de Pesquisa e Desenvolvimento; Simulação de Monte Carlo; Programação Dinâmica; avaliação de projetos; planta Gas-to-liquid. 


\section{Abstract}

Crespo, Carlos F. S.; Teixeira, José P. Evaluation of the Economic Impact in the Value of a "Gas-to-liquids" Facility Caused by a Research and Develeopment Tecnological Project Using the the Real Options Theory Rio de Janeiro, 2008. 128p. D.Sc Thesis - Departamento de Engenharia Industrial. Pontífícia Universidade Católica do Rio da Janeiro.

When making decisions, the figure of the manager comes across different types of uncertainties. Among them, the time to conclude a project. The characteristics of innovation and novelty in Research and Development Projects applied to technology, further reinforces the caution to be taken regarding the "time" dimension at the moment of the decision, since the penalty could culminate in the loss of a valuable business opportunity. In order to quantify the impact of the effort applied in a Research and Technological Development Project, in the value of an entrepreneurship conducted according to a context in which "time" is represented by a random variable, this study presents a pertinent model. Considering that a project sequentially evolves and can be abandoned during any stage of its development, the Theory of Real Options was the chosen theoretical framework. The model utilizes a lattice to capture the technical uncertainty, characteristics of the development of technology performance, and takes in account as a random variable the time of the conclusion of each stage of the project. The expected value of the project with the flexibility provided by the option to abandonment built in the Research and Development Project, is calculated through a combination of the Monte Carlo Simulation and the technique of Dynamic Programming. The proposed model was applied in the case of a Gas-to-liquid (GTL) plant. The payoff function of the Research and Development Project was built through several contributions stemmed from an agreement between the Industrial Engineering Department of PUC-RJ and Petrobras S.A., in order to assess the economic value of a GTL plant.

\section{Key Words}

Real Options; Research and Development Project; Monte Carlo Simulation; Dynamic Programming; Project Valuation; Gas to Liquid Plant 


\section{Sumário}

1 Introdução 16

$\begin{array}{ll}1.1 \text { Apresentação do Problema } & 16\end{array}$

$\begin{array}{ll}1.2 \text { Estruturação do Modelo } & 17\end{array}$

$\begin{array}{ll}1.3 \text { Outras Contribuições e Objetivos } & 18\end{array}$

$\begin{array}{ll}1.4 \text { Organização da Tese } & 18\end{array}$

2 Referencial Teórico 21

2.1 Introdução 21

2.2 Opções Financeiras $\quad 21$

2.3 Orçamento de Capital e a Regra do Valor Presente Líquido 22

2.4 Opções Reais (OR) 26

2.5 Opções Reais Compostas e Projetos de P\&D 28

2.6 Projetos de Pesquisa e Desenvolvimento (P\&D) 29

2.7 Incertezas de Mercado e Técnica 32

2.8 Tempo de Duração de um Projeto: Variável Aleatória 34

2.9 Pesquisa Atual 36

2.10 - Contribuições da Tese $\quad 44$

3 Processo Gas-to-liquids e Teoria das Opções Reais 46

3.1 O Processo Gas-To-Liquids 46

3.2 Estudos de Viabilidade Econômica de Projetos GTL 50

3.2.1 Abordagem pela Metodologia do Valor Presente Líquido 50

3.2.2 Abordagem Opcional 52

3.3 Pesquisa e Desenvolvimento em Tecnologia Gas-to-Liquids 54

4 Modelo de Apoio à Decisão Voltado para o Produto 58

4.1 Huchzermeier \& Loch 59

4.2 Santiago \& Vakili 63

5 O Modelo Voltado para a Tecnologia 66

5.1 Apresentação do Modelo 66

5.2 A Função "Payoff" 
5.3 Redução do "CAPEX"

5.4 Faixa Relevante da Função "Payoff"

5.5 A Introdução do Tempo na Função "Payoff" 75

5.6 Processo de Cálculo do Valor da Flexibilidade do

Projeto de P\&D $\quad 77$

6 Resultados da Aplicação do Modelo 78

6.1 Introdução $\quad 78$

6.2 Avaliação do Desempenho da Tecnologia 78

6.3 Flexibilidade Gerencial e Custos 79

6.4 A Função "Payoff" 80

6.4.1 Relação Eficiência e "CAPEX" da Planta 80

6.4.2 Tempo 81

6.5 Cálculo do Valor da Flexibilidade 83

6.6 Análise de Sensibilidade $\quad 85$

6.7 Resultados $\quad 86$

6.7.1 Variância 86

6.7.2 Valor Esperado 90

6.7.3 Custos Variáveis 93

6.7.4 Taxa de Juros $\quad 96$

6.7.5 Comparações do comportamento das flexibilidades para as distribuições Uniforme e Triangular 99

6.7.5.1 Média Constante - Desvio Padrão Crescente 100

6.7.5.2 Média crescente - Desvio Padrão constante 102

6.7.5.3 Breve Comentário Sobre a Comparação do

Comportamento das Distribuições Uniforme e Triangular 104

7 Conclusões e Sugestões 105

8 Referências Bibliográficas 109

Apêndice A: Simulação de Monte Carlo 115

Apêndice B: Equação de Distribuição dos Produtos

da Síntese F\&T 
Apêndice C: Perfis de Produção para Diversos valores de $\alpha \quad 118$

Apêndice D: Programação Dinâmica 119

Apêndice E: Um Breve Exemplo do Modelo $\quad 120$

Apêndice F: Dados de Entrada (em vermelho) da

Planilha de Francisco [7] 124

Apêndice G: Dados de Entrada para os Processos

Estocásticos dos Preços dos “input”s e "output”s da Planta GTL 125

Apêndice H: llustração da Função Payoff 126

Apêndice I: Planilhas de Cálculo para as Distribuições T e U 128 


\section{Lista de figuras}

Figura 3.1 - Esquema Básico do Processo Gas-to-Liquids 48

Figura 4.1 - Probabilidades de Transição - Exemplo 60

Figura 4.2 - Probabilidades de Transição, com Deslocamento - Exemplo 61

Figura 4.3 - Modelo Huchzermeier \& Loch 63

Figura 4.4 - Dinâmica do Desempenho do Produto 64

Figura 5.1 - Estágios de Desenvolvimento do Projeto de

Pesquisa e Desenvolvimento $\quad 68$

Figura 5.2 - Treliça Simulada, "Payoff” e Tempo Aleatório 71

Figura 5.3 - Faixa Relevante da Função "Payoff” 75

Figura 5.4 - Caminhos Aleatórios dos Preços e Valores da Função "Payoff" 76

Figura 6.1 - Atividades do Processo de Pesquisa e Desenvolvimento com Três Estágios $\quad 78$

Figura 6.2 - Treliça e Probabilidades de Transição 79

Figura 6.3 - Função "Payoff"

Figura 6.4 - Transição de Um Estágio para Outro 84

Figura 6.5 - Comportamento da Flexibilidade - Dist U (Variável Tempo:

Valor Esperado Constante - Desvio Padrão Aumenta) 88

Figura 6.6 - Comportamento da Flexibilidade - Dist T (Variável Tempo:

Valor Esperado Constante - Desvio Padrão Aumenta) 89

Figura 6.7 - Comportamento da Flexibilidade - Dist U (Variável Tempo:

Valor Esperado Aumenta - Desvio Padrão Constante) 92

Figura 6.8 - Comportamento da Flexibilidade - Dist T (Variável Tempo:

Valor Esperado Aumenta - Desvio Padrão Constante

Figura - 6.9 - Comportamento da Flexibilidade - Dist U (Variável

Tempo: Valor Esperado e Desvio Padrão Constantes - Custo Variável

Aumenta)

Figura 6.10 - Comportamento da Flexibilidade - Dist T (Variável

Tempo: Valor Esperado e Desvio Padrão Constantes - Custo Variável

Aumenta)

Figura 6.11 - Comportamento da Flexibilidade em Relação ao

Aumento \% da Taxa de Juros - Distribuição U

Figura 6.12 - Comportamento da Flexibilidade em Relação ao 
Aumento \% da Taxa de Juros - Distribuição T

Figura 6.13 - Comparação de Valores Gerados pelas

Distribuições Uniforme e Triangular (Média Constante -

Desvio Padrão Crescente)

101

Figura 6.14 - Comparação Valores Gerados pelas Distribuições

Uniforme e Triangular (Média Crescente - Desvio Padrão Constante) 


\section{Lista de tabelas}

Tabela 2.1- Reprodução Tabela 06 [Dias, 6] - Analogia de Uma

Opção Financeira com a Opção Real de Paddock-Siegel-Smith

Tabela 2.2 - Outras Analogias entre Opções Financeiras e Opções Reais 27

Tabela 2.3 - entre Opções Financeiras e Opções Reais [Dias, 23]

Tabela 2.4 - Algumas Conclusões dos Autores Huchzermeier \& Loch

e Santiago \& Vakili

Tabela 3.1 - Consumo Total de Energia por tipo de Combustível, 1920-2030 (BTUx10 $\left.{ }^{15}\right) \quad 46$

Tabela 3.2 - Plantas GTL em Operação 49

Tabela 3.3 - Evolução do Número de Patentes Relacionadas com a Tecnologia GTL $\quad 55$

Tabela 3.4 - Distribuição Percentual do "CAPEX" 55

Tabela 3.5 - Distribuição do "CAPEX" 56

Tabela 3.6 - Diferentes Direções de Pesquisa em GTL

e suas Características $\quad 56$

Tabela 6.1 - Relação Eficiência - "CAPEX”

Tabela 6.2 - Função "Payoff"

Tabela 6.3 - Custos (US\$), Taxa de Juros e Número de Simulações $\quad 84$

Tabela 6.4 - Parâmetros Utilizados para Construir Tabela 6.6 e Figura 6.587

Tabela 6.5 - Parâmetros Utilizados para Construir Tabela 6.7 e Figura 6.687

Tabela 6.6 - Comportamento da Flexibilidade (US\$) - Dist U (Variável

Tempo: Valor Esperado Constante - Desvio Padrão Aumenta) 88

Tabela 6.7 - Comportamento da Flexibilidade (US\$) - Dist T (Variável

Tempo: Valor Esperado Constante - Desvio Padrão Aumenta) 89

Tabela 6.8 - Parâmetros Utilizados para Construir Tabela 6.10 e Figura $6.7 \quad 90$

Tabela 6.9 - Parâmetros Utilizados para Construir Tabela 6.11 e Figura 6.891

Tabela 6.10 - Comportamento da Flexibilidade (US\$) - Dist U (Variável

Tempo: Valor Esperado Aumenta - Desvio Padrão Constante) 91

Tabela 6.11 - Comportamento da Flexibilidade (US\$) - Dist T (Variável

Tempo: Valor Esperado Aumenta - Desvio Padrão Constante) 92

Tabela 6.12 - Parâmetros Utilizados para Construir Tabela 6.14 e Figura 6.994

Tabela 6.13 - Parâmetros Utilizados para Construir Tabela 6.15 e Figura 6.1094 
Tabela 6.14 - Comportamento da Flexibilidade (US\$) - Dist U (Variável

Tempo: Valor Esperado e Desvio Padrão Constantes - Custo

Variável Aumenta)

Tabela 6.15 - Comportamento da Flexibilidade (US\$) - Dist T (Variável

Tempo: Valor Esperado e Desvio Padrão Constantes - Custo

Variável Aumenta)

Tabela 6.16 - Cálculo do Valor da Flexibilidade (US\$) para Diversas

Taxas de Juros

Tabela 6.17 - Comportamento da Flexibilidade (US\$) em Relação ao

Aumento \% da Taxa de Juros - Distribuição U

Tabela 6.18 - Comportamento da Flexibilidade (US\$) em Relação ao

Aumento \% da Taxa de Juros - Distribuição T

Tabela 6.19 - Parâmetros Utilizados para Construir Tabela 6.21 e

Figura 6.13 (Distribuição U)

Tabela 6.20 - Parâmetros Utilizados para Construir Tabela 6.21 e

Figura 6.13 (Distribuição T)

100

Tabela 6.21 - Comparação de Valores (US\$) Gerados pelas

Distribuições Uniforme e Triangular (Média Constante - Desvio

Padrão Crescente)

101

Tabela 6.22 - Parâmetros Utilizados para Construir Tabela 6.24 e

Figura 6.14 (Distribuição U)

Tabela 6.23 - Parâmetros Utilizados para Construir Tabela 6.24 e

Figura 6.14 (Distribuição T)

Tabela 6.24 - Comparação Valores (US\$) Gerados pelas

Distribuições Uniforme e Triangular (Média Crescente - Desvio

Padrão Constante $=0,0612$ anos) 


\section{Lista de siglas e abreviaturas}

GTL - Gas-to liquid

P\&D - Pesquisa e Desenvolvimento

FCD - Fluxo de caixa Descontado

EVTE - Estudo de Viabilidade Técnica e Econômica

VPL - Valor Presente Líquido

F\&T - Fischer e Tropsch

OR - Opções Reais

U - Distribuição Uniforme de Probabilidades

$\mathrm{T}$ - Distribuição Triangular de Probabilidades

BTU - British Thermal Unit

ASF - Anderson-Schulz-Flory 